\title{
GLIPR1 inhibits the proliferation and induces the differentiation of cancer-initiating cells by regulating miR-16 in osteosarcoma
}

\author{
JIAN DONG $^{1}$, BINNA BI ${ }^{1}$, LIANHAI ZHANG ${ }^{2}$ and KAITUO GAO ${ }^{1}$ \\ ${ }^{1}$ Department of Orthopedics, Linyi People's Hospital, Linyi, Shandong 276003; \\ ${ }^{2}$ Department of Emergency Surgery, Zaozhuang Municipal Hospital, Zaozhuang, Shandong 277102, P.R. China
}

Received February 19, 2016; Accepted March 30, 2016

DOI: $10.3892 /$ or.2016.4949

\begin{abstract}
Osteosarcoma is a common, highly malignant and metastatic bone cancer. Elucidation of the molecular mechanisms of osteosarcoma may further help us to understand the pathogenesis of the disease, and offer novel targets for effective therapies. Human glioma pathogenesis-related protein 1 (GLIPR1) has been found to be downregulated in human cancers. However, its roles have not been reported in osteosarcoma. In the present study, we demonstrated that GLIPR1 protein was downregulated in osteosarcoma. Its overexpression inhibited the proliferation, migration and invasion and induced the differentiation of cancer-initiating cells (CICs) in osteosarcoma. Moreover, GLIPR1 overexpression upregulated miR-16 in osteosarcoma cells. The upregulation suppressed proliferation, migration and invasion as well as induced differentiation of CICs in osteosarcoma. Thus, we conclude that GLIPR1 inhibited the proliferation, migration and invasion and induced the differentiation of CICs by regulating miR-16 in osteosarcoma. The present study provides direct evidence that GLIPR1 is a bona fide tumor suppressor and identified GLIPR1 and miR-16 as key components for regulating the proliferation, migration, invasion and CICs in osteosarcoma.
\end{abstract}

\section{Introduction}

Osteosarcoma is a malignant neoplasm of the bone that is prevalent in teenagers and young adults. Although it has been reported that numerous factors are associated with the increased risk of osteosarcoma including age, genetic inheritance, chronic inflammation, viral infection and radiation exposure, the cause of osteosarcoma remains undetermined (1). Most osteosarcoma patients are treated with chemotherapy or radiation therapy. Yet, some patients remain at high risk of relapse or metastasis, thus necessitating a successful treatment strategy (2). Since osteosarcoma has highly invasive and metastatic potential,

Correspondence to: Dr Kaituo Gao, Department of Orthopedics, Linyi People's Hospital, 48 Jiefang Road, Lanshan, Linyi, Shandong 276003, P.R. China

E-mail: gaokaituo03@126.com

Key words: osteosarcoma, GLIPR1, miR-16 determining the factors promoting survival, migration and invasion of osteosarcoma cells is imperative $(3,4)$. Elucidation of the molecular mechanisms involved in the promotion of proliferation, migration and invasion in osteosarcoma may further aid in the understanding of the pathogenesis of the disease, but also may offer novel targets for effective therapies.

Human glioma pathogenesis-related protein 1 (GLIPRI), also known as CRISP7, is a p53 target gene which is downregulated in cancer and one of the reasons for its deregulation is due to methylation of its promoter (5-8). Lack of GLIPR1 was found to be associated with reduced tumor-free survival in an animal model, and the orthotopic injection of adenoviral vectors overexpressing GLIPR1 in a mouse model of metastatic prostate cancer led to decreased microvessel density, implying that GLIPR1 has anti-angiogenic ability and can increase infiltration of cancer-associated macrophages and cytotoxic T cells (9-13). These studies indicated that GLIPR1 is a tumor-suppressor. However, an understanding of the regulatory mechanisms of GLIPR1 remain incomplete.

MicroRNAs (miRNAs/miRs) are regulatory, non-coding RNAs $\sim 18-25$ nucleotides in length and are expressed at specific stages of tissue development or cell differentiation. They have large-scale effects on the expression of a variety of genes at the post-transcriptional level (14-19). The discovery of miRNAs has broadened our scope and understanding of the mechanisms that can regulate gene expression (14-19). miRNAs induce mRNA degradation or translational suppression through base-pairing with its targeted mRNAs (14-19). The aberrant expression of miRNAs has been linked to various human types of cancer and has been proposed to have an oncogenic or a tumor-suppressor role and they have been shown to play key roles in cell survival, proliferation, apoptosis, migration, invasion, angiogenesis and various other characteristic features which are altered in cancers $(20,21)$.

Recently, it has been reported that miR-16 is downregulated in osteosarcoma cell lines and tissues (22). Overexpression of miR-16 was found to suppress proliferation and tumor growth in an animal model of osteosarcoma (22). Furthermore, IGF1R, as a tumor-suppressive gene, is a direct target of miR-16 and its expression is inversely correlated with miR-16 levels in osteosarcoma (22). Mechanistic investigation further revealed that miR-16 overexpression inhibited the Raf1/MEK1/2/ERK pathway. Yet, the identification of novel target genes of miR-16 may help us to further understand its role in osteosarcoma. 
In the present study, we showed that GLIPR 1 protein was downregulated in osteosarcoma. Its overexpression inhibited proliferation, migration and invasion and induced differentiation of cancer-initiating cells (CICs) in osteosarcoma. Moreover, GLIPR1 overexpression upregulated miR-16 expression in osteosarcoma cells. The upregulation suppressed the proliferation, migration and invasion as well as induced differentiation of CICs in osteosarcoma. Thus, we conclude that GLIPR1 inhibited the proliferation, migration and invasion and induced the differentiation of CICs by regulating miR-16 in osteosarcoma. The present study provides direct evidence that GLIPR1 is a bona fide tumor suppressor and identifies GLIPR1 and miR-16 as key components for regulating the proliferation, migration, invasion and CICs in osteosarcoma.

\section{Materials and methods}

Osteosarcoma tissues. Osteosarcoma and adjacent normal tissues were obtained from the Department of Orthopedics, Linyi People's Hospital Affiliated to Shandong University, Linyi, Shandong. All tissues were histologically examined, and pathologists confirmed the diagnosis. The Huabei Medical Ethics Committee of Linyi People's Hospital approved the experiments undertaken. The use of the human tissue samples followed internationally recognized guidelines as well as local and national regulations. Informed consent was obtained from each individual.

Cell culture. Osteosarcoma cell line MG63 was obtained from the American Type Culture Collection (ATCC; Vanassas, MA, USA). Briefly, the cells were maintained in RPMI-1640 medium supplemented with $10 \%$ fetal bovine serum (FBS) (Gibco, Grand Island, NY, USA) and penicillin/streptomycin at $37^{\circ} \mathrm{C}$ in a humidified atmosphere with $5 \% \mathrm{CO}_{2}$.

Cell transfection. All expression plasmids were purchased from Tiangen (Tianjin, China). miR-16/miR controls were purchased from Ambion, Inc. (Ambion, Austin, TX, USA). Cells were seeded into 6 -well plates $24 \mathrm{~h}$ before transfection. When the cells reached $80 \%$ confluency, the expression plasmids were transfected into the MG63 cells using Lipofectamine 2000 (Invitrogen, Carlsbad, CA, USA) according to the manufacturer's instructions.

Western blot analysis. Western blot analysis was performed as previously described (22), mainly after incubation with the primary antibodies anti-GLIPR1 (1:250); anti-CD133 (1:250); anti-Nestin (1:250) and anti- $\beta$-actin (1:500) (all from Abcam, Cambridge, MA, USA) overnight at $4^{\circ} \mathrm{C}$. IRDye ${ }^{\mathrm{TM}} 800$-conjugated anti-rabbit secondary antibody (LI-COR, Biosciences, Lincoln, NE, USA) was used for $30 \mathrm{~min}$ at room temperature. The specific proteins were visualized by Odyssey ${ }^{\mathrm{TM}}$ Infrared Imaging System (Gene Company, Lincoln, NE, USA).

Cell proliferation. The effect on the cell proliferation was assessed using the 3-(4,5-dimethylthiazol-2-yl)-2,5-diphenyltetrazolium bromide (MTT; Sigma, St. Louis, MO, USA) assay and it was performed as previously described (23). Absorbance was directly proportional to the number of surviving cells.
Flow cytometric analysis. CD133 and Nestin expression analyses were performed by flow cytometric analysis according to the instructions. Briefly, the cells were dissociated into single-cell populations and labeled with a phycoerythrinconjugated CD133 or Nestin antibody (Abcam). The expression level was calculated using the EPICS XL flow cytometer with EXPO32 ADC software (Becton-Dickinson, San Jose, CA, USA).

BrdU incorporation assay. The BrdU incorporation assay was performed as previously described (22). Cells grown on coverslips (Fisher, Pittsburgh, PA, USA) were incubated with bromodeoxyuridine (BrdU) for $1 \mathrm{~h}$ and stained with the antiBrdU antibody (Upstate, Temecula, CA, USA) according to the manufacturer's instructions. Images were captured under a laser scanning microscope (Axioskop 2 plus; Carl Zeiss Co., Ltd., Jena, Germany).

Colony formation assay. Colony formation assay was performed as previously described (22). For the colony formation assay, cells were transfected as indicated, and then seeded into a 6 -well plate. FBS was added $(0.3 \mathrm{ml} /$ well $)$ on day 5. After 9 days of incubation, the plates were washed with phosphate-buffered saline (PBS) and stained with $0.1 \%$ crystal violet. Colonies with over 50 cells were manually counted.

Real-time PCR for miRNAs. Total RNA from the cultured cells, with efficient recovery of small RNAs, was isolated using the mirVana miRNA isolation kit (cat no. AM2654; Ambion, Inc.). Detection of the mature form of miRNAs was performed using the mirVana qRT-PCR miRNA Detection kit (cat. no. AM7659; Ambion, Inc.), according to the manufacturer's instructions. The U6 small nuclear RNA was used as an internal control.

Sphere growth. Osteosarcoma cells $\left(1 \times 10^{3} / \mathrm{ml}\right)$ in serum-free RPMI-1640/1 mM Na pyruvate were seeded on $0.5 \%$ agar precoated 6-well plates. After 7 days, one third of the medium was exchanged every second day. Single spheres were chosen and counted.

Invasion and wound healing assays, and miRNA detection. Invasion and wound healing assays, and miRNA detection were performed as previously described (24).

Statistical analysis. Data are presented as the mean \pm SEM. Student's t-test (two-tailed) was used to compare two groups $(\mathrm{P}<0.05$ was considered to indicate a statistically significant result).

\section{Results}

GLIPR1 protein is downregulated in osteosarcoma. In an attempt to identify GLIPR1 expression between osteosarcoma and adjacent normal tissues, we performed western blotting in osteosarcoma tissues vs. normal tissues. Protein was isolated from 6 pairs of osteosarcoma and normal tissues (patients no. 1-6). We found that GLIPR1 protein was significantly decreased in the sarcoma tissues, compared with that noted in 

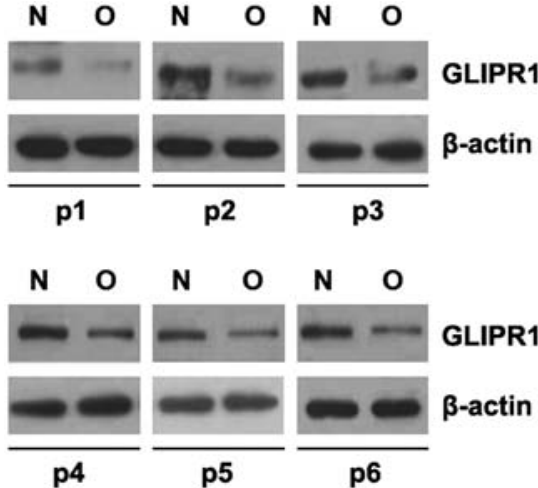

Figure 1. GLIPR1 is downregulated in osteosarcoma. Western blotting of GLIPR1 protein in osteosarcoma $(\mathrm{O})$ and adjacent normal tissues $(\mathrm{N})$ from 6 patients (p). $\beta$-actin was used as a loading control. $n=6$.

the adjacent normal tissues (Fig. 1). This implies that GLIPR1 could be a tumor-suppressor gene in osteosarcoma.

GLIPRI inhibits proliferation, migration and invasion in osteosarcoma. To investigate whether GLIPR1 can affect the proliferation of osteosarcoma cells, firstly using western blotting, we tested whether GLIPR1-expressing plasmids could stably express GLIPR1 protein in MG63 cells. The results showed that GLIPR1 protein was significantly increased by the GLIPR1-expressing plasmids in the cells (Fig. 2A). In addition, we performed MTT assay to detect proliferation of the MG63 cells following transfection with the GLIPR1-expressing plasmids. The results showed that GLIPR1 inhibited the proliferation of the MG63 cells after $48 \mathrm{~h}$ of transfection (Fig. 2B). To further study the effects of GLIPR1 on proliferation, we performed BrdU incorporation assay to detect DNA synthesis in the cells. The results confirmed that GLIPR1 significantly inhibited DNA synthesis in the cells (Fig. 2C). In order to identify the effect of GLIPR1 on colony formation, we performed a colony formation assay. The results showed that overexpression of GLIPR1 significantly suppressed the colony formation rate of the MG63 cells following transfection (Fig. 2D).

In an attempt to identify the role of GLIPR1 in regulating invasion and migration of MG63 cells, we performed invasion and would healing assays to detect the invasion and migration of the MG63 cells following transfection with the GLIPR1-expressing plasmids and empty vectors. Ectopic GLIPR1 did inhibit the invasion and motility by $\sim 2$-fold in the cells (Fig. 2E and F).

GLIPRI induces differentiation of CICs in osteosarcoma. Malignant tumors tend to relapse after surgical resection, and this behavior is believed to be largely attributable to the stem cell-like properties of a fraction of cells (25). Since GLIPRI is expressed at very low levels in osteosarcoma, but at high levels in differentiated normal tissues, we aimed to determine the potential role of GLIPRI in the development and maintenance of the stem-like property of osteosarcoma cells. A sphere forming assay showed (Fig. 3A) that GLIPRI-overexpressing cells formed much smaller spheres after 7 days of culture when compared with the control cells ( 2 -fold smaller in diameter), indicating markedly decreased self-renewal ability by GLIPR1.
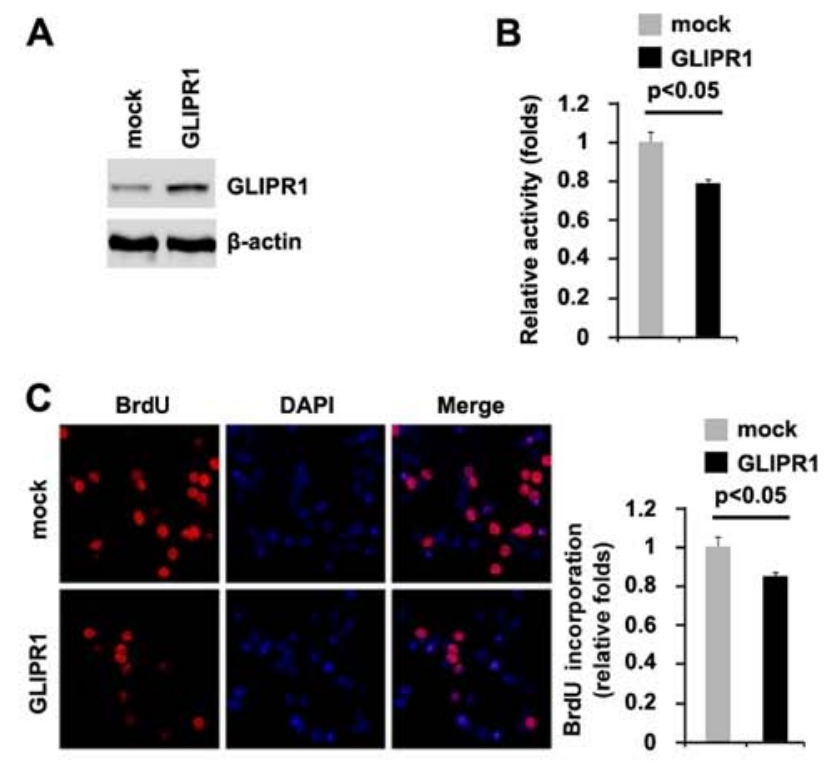

D
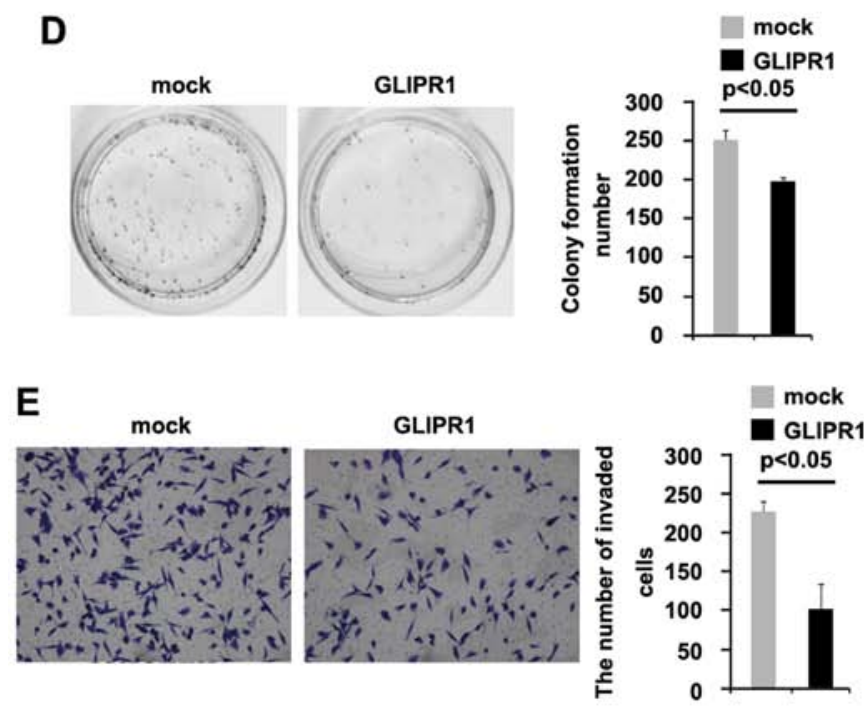

$\mathbf{F}$
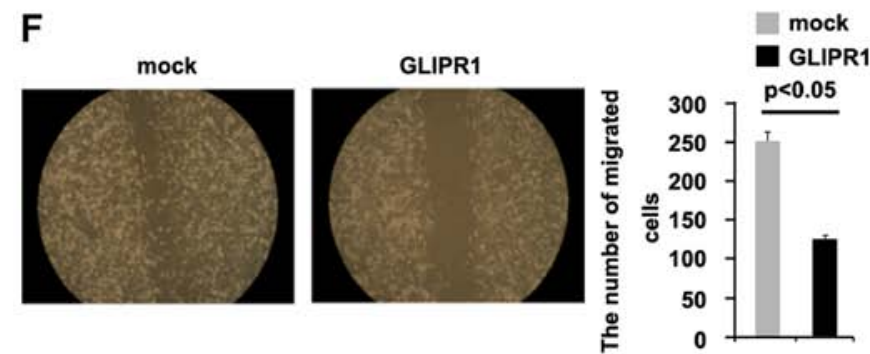

Figure 2. GLIPR1 inhibits proliferation, motility and invasion in osteosarcoma. (A) Western blotting of GLIPR1 in MG63 cells. MG63 cells were transfected with GLIPR1-expressing plasmids or empty vectors (mock). $\beta$-actin was used as a loading control; $n=3$. (B) MTT assay of MG63 cells. MG63 cells were transfected with GLIPR1-expressing plasmids or empty vectors; $n=3$. (C) BrdU incorporation assay of MG63 cells. Cells were transfected with GLIPR1-expressing plasmids or empty vectors (mock). Left panel shows microscopic images of fluorescence staining of one representative experiment (magnification, $x 100$ ). Right panel shows graphic presentation of mean fluorescence intensities; $n=3$. (D) Colony formation assay of MG63 cells transfected with GLIPR1-expressing plasmids or empty vectors (mock). Colonies with over 50 cells were counted. Representative micrographs (left) and quantification of colonies (right) after transfection with GLIPR1 expressing plasmids or empty vectors (mock); $n=3$. (E) Invasion assays of MG63 cells transfected with GLIPR1 and empty vectors (mock). (F) Wound-healing assays of MG63 cells transfected with GLIPR1 and empty vectors (mock). The cell layer was photographed; $\mathrm{n}=3$. 

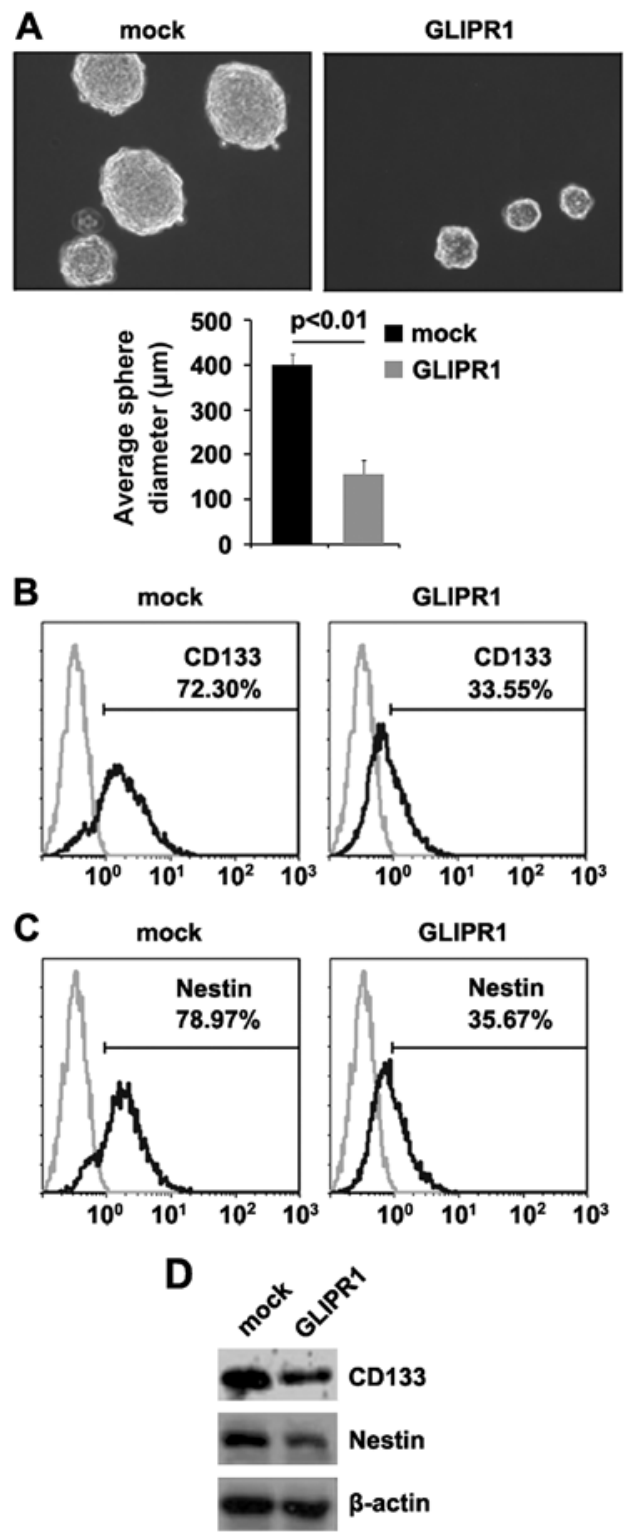

Figure 3. GLIPR1 induces the differentiation of cancer-initiating cells in osteosarcoma. (A) Sphere growth of MG63 cells transfected as indicated. Upper panel, representative images of spheres formed by the indicated cells after 7 days of culture. Lower panel, statistical analysis of the average diameter of the spheres. (B) FACS for CD133 in sphere-derived cells $48 \mathrm{~h}$ after transfection. (C) FACS for Nestin in sphere-derived cells $48 \mathrm{~h}$ after transfection. (D) Western blotting for CD133 and Nestin in the MG63 cells transfected with GLIPR1-expressing plasmids or empty vectors (mock) $\beta$-actin was used as a loading control; $n=3$.

Consistent with these results, CD133- and Nestin-positive cell proportions were significantly lower in the GLIPRItransfected cells than those in the control cells (Fig. 3B and C). In further experiments, we performed western blotting to detect CD133 and Nestin protein in the MG63 cells following transfection with the GLIPR1-expressing plasmids and empty vectors. We observed a significantly faster decrease in CD133 and Nestin protein in the MG63 cells transfected with GLIPR1 (Fig. 3D). Taken together, these data showed that reintroduction of GLIPRI in osteosarcoma cells reduced the stem cell-like population and greatly attenuated the ability of stem cell-like osteosarcoma cells to retain stemness.
GLIPRI upregulates miR-16 expression and miR-16 suppresses the proliferation, migration and invasion in osteosarcoma. Tumor-suppressor genes can exert their functions by regulating miRNA expression in cancer (26) and in regards to the miRNA involvement in lung cancer pathogenesis, some of them function as tumor-suppressor genes or oncogenes $(27,28)$. Thus, we reasoned that GLIPR1 functions as a tumor-suppressor gene by regulating relevant miRNAs. miRNA microarray was performed. RNAs isolated from the MG63 cells transfected with GLIPR1 or empty vectors were hybridized to a custom miRNA microarray platform. After three times of hybridization, quantification and normalization, 200 miRNAs were upregulated $>5$-fold in the cells. We were interested in miR-16 (Fig. 4A), since it is downregulated in osteosarcoma indicating that it may be a tumor-suppressor gene.

To further identify whether GLIPR1 upregulates the miR-16 level in MG63 cells, northern blotting was performed. Our results demonstrated that miR-16 was upregulated by GLIPR1 in the MG63 cells (Fig. 4B).

To investigate whether miR-16 can affect the proliferation of osteosarcoma cells, firstly using real-time PCR, we tested whether pre-miR-16 stably expressed miR-16 in the MG63 cells. The results showed that miR-16 was significantly increased by pre-miR-16 in the cells (Fig. 4C). In addition, we performed MTT assay to detect the proliferation of MG63 cells following transfection with pre-miR-16. The results showed that miR-16 inhibited the proliferation in MG63 cells after $48 \mathrm{~h}$ of transfection (Fig. 4D). To further show the effects of miR-16 on proliferation, we performed BrdU incorporation assay to detect DNA synthesis in the cells. The results confirmed that miR-16 significantly inhibited DNA synthesis in the cells (Fig. 4E). In an attempt to identify the role of miR-16 in regulating migration and invasion of MG63 cells, we performed wound healing and invasion assays to detect the migration and invasion of MG63 cells following transfection with pre-miR-16 and control miR. Ectopic miR-16 inhibited the motility and invasion by $\sim 2$-fold in the cells (Fig. 2F and G).

miR-16 induces the differentiation of CICs in osteosarcoma. Since GLIPR1 induces the differentiation of CICs and it upregulates miR-16 expression, we aimed to determine the potential role of $m i R-16$ in the development and maintenance of the stem-like properties of osteosarcoma cells. Sphere-forming assay showed (Fig. 5A) that $m i R$-16-overexpressing cells formed much smaller spheres after 7 days of culture as compared with the control cells ( $\sim 2$-fold smaller in diameter), indicating the markedly decreased self-renewal ability by miR- 16 .

Consistent with these results, CD133- and Nestin-positive proportions were significantly lower in the $m i R$-16-expressing cells than levels in the control cells (Fig. 5B and C). In further experiments, we performed western blotting to detect CD133 and Nestin protein in the MG63 cells following transfection with pre-miR-16 and miR control. We also observed a significantly faster decrease in CD133 and Nestin protein in the MG63 cells transfected with pre-miR-16 (Fig. 5D). Taken together, these data showed that reintroduction of miR-16 in the osteosarcoma cells reduced the stem cell-like population and greatly attenuated the ability of stem cell-like osteosarcoma cells to retain stemness. 

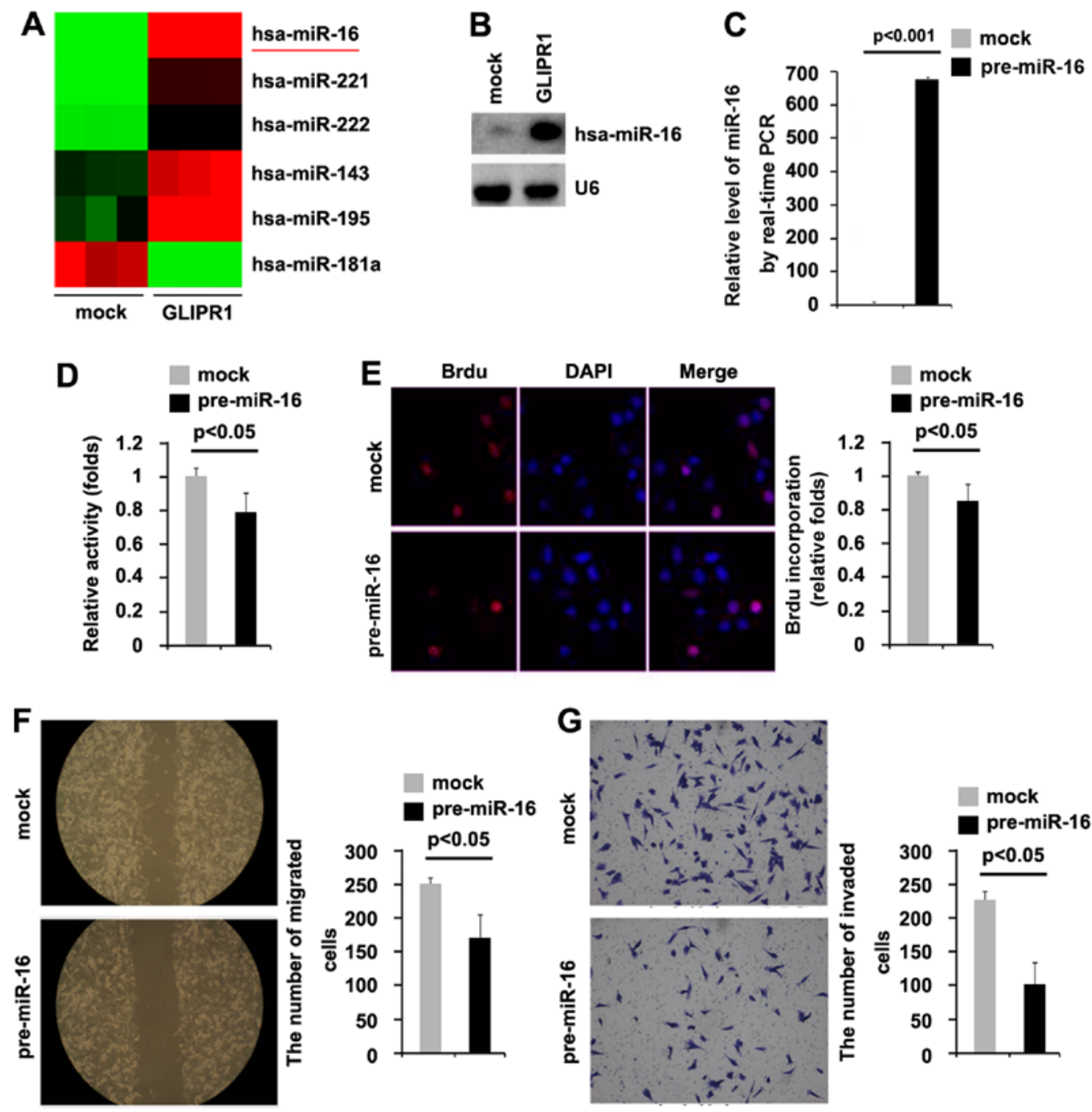

Figure 4. GLIPR1 upregulates miR-16 expression and miR-16 inhibits proliferation, motility and invasion in osteosarcoma. (A) miRNA microarray analysis of MG63 cells transfected with GLIPR1-expressing plasmids or empty vectors (mock); n=3. (B) Northern blotting for miR-16 in MG63 cells transfected with GLIPR1-expressing plasmids or empty vectors (mock).U6 was used as a loading control; $n=3$. (C) Real-time RT-PCR for miR-16 in MG63 cells transfected with pre-miR-16 or control miR (mock). U6 was used as a loading control; n=3. (D) MTT assay of MG63 cells. MG63 cells were transfected with pre-miR-16 or control miR (mock); $n=3$. (E) BrdU incorporation assay of MG63 cells. Cells were transfected with pre-miR-16 or control miR (mock). Left panel shows microscopic images of fluorescence staining of one representative experiment (magnification, x100). Right panel shows graphic presentation of mean fluorescence intensities; $n=3$. (F) Wound-healing assays of MG63 cells transfected with pre-miR-16 and control miR (mock). The cell layer was photographed; $n=3$. (G) Invasion assays of MG63 cells transfected with pre-miR-16 and control miR (mock).

\section{Discussion}

Recent studies have indicated that solid tumors, including osteosarcoma, are driven by a population of tumor stem cells (TSCs) or tumor-initiating cells (TICs) $(29,30)$. It is believed that TSCs can fuel tumor growth and seed metastasis. Although conventional chemotherapy can target proliferating tumor cells and it is common to find tumor regression, some osteosarcoma patients develop tumor relapse or metastasis after chemotherapy and surgery. This clinical clue allows us to presume that current approaches are not efficient to target TSCs, according to the concept of TSCs $(31,32)$.

Thus, finding novel targets for eliminating TSCs is vital. The present study showed that GLIPR1 protein is downregulated in osteosarcoma and GLIPR 1 inhibits proliferation, migration and invasion in osteosarcoma, meanwhile inducing differentiation of cancer-initiating cells (CICs) in osteosarcoma (Fig. 6).
CSCs have been shown to be more resistant to standard chemotherapeutic agents and radiotherapy. Traditional treatment may lead to tumor shrinkage, however most tumors can recur after treatment, likely since CSCs survive and regenerate tumor growth $(33,34)$. Treatment strategies to restore GLIPR1 protein has the potential to be an effective therapy for osteosarcoma.

A large body of evidence indicates that miRNAs are frequently deregulated in a variety of human malignancies (35). Studies have shown a direct link between miRNA function and oncogenesis which is supported by examining the expression of miRNAs in clinical samples $(36,37)$. The profiling of miRNA expression showed that most of them are downregulated in tumors compared to normal tissues (38), such as let-7 in lung cancers (39) and miR-127 in human bladder cancers (40). However, there are other miRNAs which are upregulated in tumors, such as miR-150 in gastric 
A

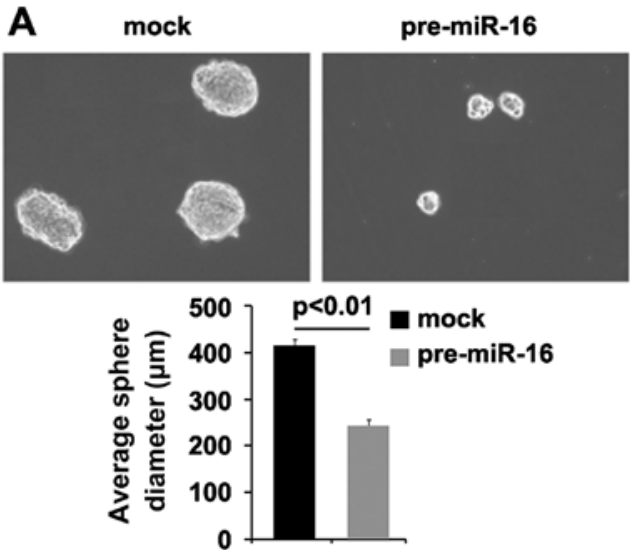

B
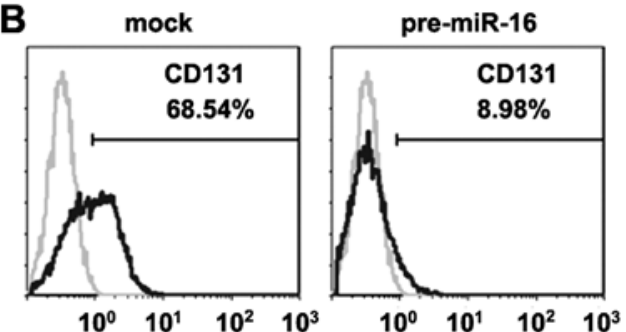

C
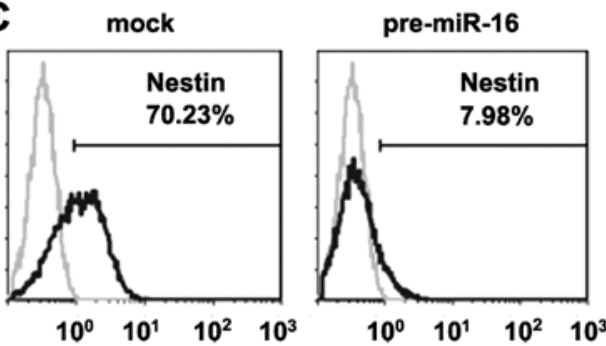

D

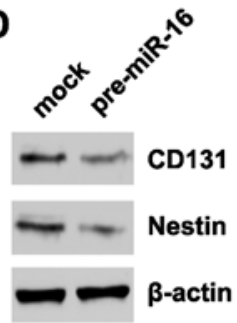

Figure 5. (A) miR-16 induces differentiation of cancer-initiating cells in osteosarcoma cells. Sphere growth of MG63 cells transfected as indicated. Upper panel, representative images of spheres after 7 days of culture. Lower panel, statistical analysis of the average diameter of spheres. (B) FACS for CD133 in sphere-derived cells $48 \mathrm{~h}$ after transfection. (C) FACS for Nestin in sphere-derived cells $48 \mathrm{~h}$ after transfection. (D) Western blotting for CD133 and Nestin in MG63 cells transfected with pre-miR-16 or control miR (mock). $\beta$-actin was used as a loading control; $n=3$.

cancer (41), miR-21 in prostate cancer (42) and the miR-17-92 cluster in renal cell carcinoma (43). We focused on miR-16 since previous studies demonstrated that the expression of miR-16 was significantly decreased in primary osteosarcoma samples as compared with adjacent normal tissues (22). We showed that miR-16 inhibited the proliferation, motility and invasion in osteosarcoma, meanwhile inducing differentiation of CICs in osteosarcoma cells (Fig. 6).

In addition, we demonstrated that GLIPR1 can upregulate miR-16 expression in ostesosarcoma cells (Fig. 6). In the future, we may continue to analyze whether the GLIPR1 protein level

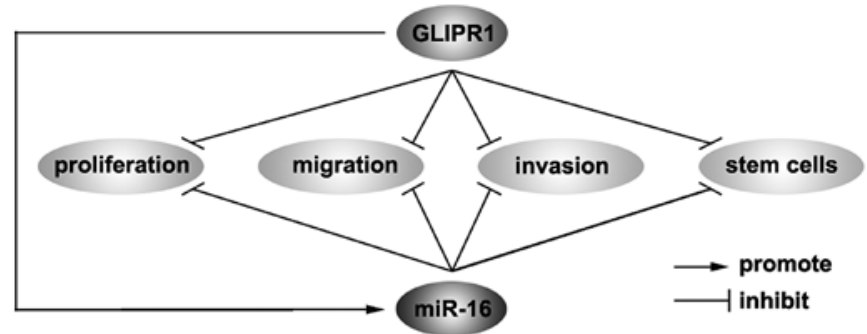

Figure 6. Diagram showing that GLIPR1 inhibits the proliferation, motility, invasion and induces differentiation of cancer-initiating cells by regulating miR-16 in osteosarcoma.

is positively associated with miR-16 expression in osteosarcoma tissues and to detect whether GLIPR1 upregulates the miR-16 level by activating its promoter.

In the present study, GLIPR1-mediated miR-16 regulation in osteosarcoma has potential basic and clinical implications (Fig. 6). On the one hand, GLIPR1 could be a powerful tumor-suppressor gene by promoting proliferation, motility and invasion as well as regulating osteosarcoma stem cells and pharmacological inhibition of GLIPR1 may represent a promising therapeutic strategy. On the other hand, miR-16 is a tumor-suppressor gene and its expression is promoted by GLIPR1 in osteosarcoma. Yet, the role of GLIPR1-mediated miR-16 regulation in osteosarcoma warrants further investigation. We will continue to identify downstream target genes of miR-16 in osteosarcoma.

\section{References}

1. Ottaviani G and Jaffe N: The etiology of osteosarcoma. Cancer Treat Res 152: 15-32, 2009.

2. Wang SW, Wu HH, Liu SC, Wang PC, Ou WC, Chou WY, Shen YS and Tang CH: CCL5 and CCR5 interaction promotes cell motility in human osteosarcoma. PLoS One 7: e35101, 2012.

3. Gill J, Ahluwalia MK, Geller D and Gorlick R: New targets and approaches in osteosarcoma. Pharmacol Ther 137: 89-99, 2013.

4. Bjørnland K, Flatmark K, Pettersen S, Aaasen AO, Fodstad O and Maelandsmo GM: Matrix metalloproteinases participate in osteosarcoma invasion. J Surg Res 127: 151-156, 2005.

5. Ren C, Li L, Goltsov AA, Timme TL, Tahir SA, Wang J, Garza L, Chinault AC and Thompson TC: $m R T V P-1$, a novel p53 target gene with proapoptotic activities. Mol Cell Biol 22: 3345-3357, 2002.

6. Ren C, Li L, Yang G, Timme TL, Goltsov A, Ren C, Ji X, Addai J, Luo H, Ittmann MM, et al: RTVP-1, a tumor suppressor inactivated by methylation in prostate cancer. Cancer Res 64: 969-976, 2004.

7. Xiao YH, Li XH, Tan T, Liang T, Yi H, Li MY, Zeng GQ, Wan XX, Qu JQ, He QY, et al: Identification of GLIPR1 tumor suppressor as methylation-silenced gene in acute myeloid leukemia by microarray analysis. J Cancer Res Clin Oncol 137: 1831-1840, 2011.

8. Chilukamarri L, Hancock AL, Malik S, Zabkiewicz J, Baker JA, Greenhough A, Dallosso AR, Huang TH, Royer-Pokora B, Brown $\mathrm{KW}$, et al: Hypomethylation and aberrant expression of the glioma pathogenesis-related 1 gene in Wilms tumors. Neoplasia 9: 970-978, 2007.

9. Li L, Abdel Fattah E, Cao G, Ren C, Yang G, Goltsov AA, Chinault AC, Cai WW, Timme TL and Thompson TC: Glioma pathogenesis-related protein 1 exerts tumor suppressor activities through proapoptotic reactive oxygen species-c-Jun- $\mathrm{NH}_{2}$ kinase signaling. Cancer Res 68: 434-443, 2008.

10. Satoh T, Timme TL, Saika T, Ebara S, Yang G, Wang J, Ren C, Kusaka N, Mouraviev V and Thompson TC: Adenoviral vectormediated mRTVP-1 gene therapy for prostate cancer. Hum Gene Ther 14: 91-101, 2003. 
11. Sonpavde G, Thompson TC, Jain RK, Ayala GE, Kurosaka S, Edamura K, Tabata K, Ren C, Goltsov AA, Mims MP, et al: GLIPR1 tumor suppressor gene expressed by adenoviral vector as neoadjuvant intraprostatic injection for localized intermediate or high-risk prostate cancer preceding radical prostatectomy. Clin Cancer Res 17: 7174-7182, 2011.

12. Naruishi K, Timme TL, Kusaka N, Fujita T, Yang G, Goltsov A, Satoh T, Ji X, Tian W, Abdelfattah E, et al: Adenoviral vectormediated RTVP-1 gene-modified tumor cell-based vaccine suppresses the development of experimental prostate cancer. Cancer Gene Ther 13: 658-663, 2006.

13. Karantanos T, Tanimoto R, Edamura K, Hirayama T, Yang G, Golstov AA, Wang J, Kurosaka S, Park S and Thompson TC: Systemic GLIPR1- $\Delta \mathrm{TM}$ protein as a novel therapeutic approach for prostate cancer. Int J Cancer 134: 2003-2013, 2014.

14. Bartel DP: MicroRNAs: Genomics, biogenesis, mechanism, and function. Cell 116: 281-297, 2004.

15. Lee RC, Feinbaum RL and Ambros V: The C. elegans heterochronic gene lin-4 encodes small RNAs with antisense complementarity to lin-14. Cell 75: 843-854, 1993.

16. Pasquinelli AE, Reinhart BJ, Slack F, Martindale MQ, Kuroda MI, Maller B, Hayward DC, Ball EE, Degnan B, Müller P, et al: Conservation of the sequence and temporal expression of let-7 heterochronic regulatory RNA. Nature 408: 86-89, 2000.

17. Reinhart BJ, Slack FJ, Basson M, Pasquinelli AE, Bettinger JC Rougvie AE, Horvitz HR and Ruvkun G: The 21-nucleotide let-7 RNA regulates developmental timing in Caenorhabditis elegans. Nature 403: 901-906, 2000.

18. Lewis BP, Burge CB and Bartel DP: Conserved seed pairing, often flanked by adenosines, indicates that thousands of human genes are microRNA targets. Cell 120: 15-20, 2005.

19. Farh KK, Grimson A, Jan C, Lewis BP, Johnston WK, Lim LP, Burge CB and Bartel DP: The widespread impact of mammalian microRNAs on mRNA repression and evolution. Science 310 1817-1821, 2005

20. Calin GA and Croce CM: MicroRNA-cancer connection: The beginning of a new tale. Cancer Res 66: 7390-7394, 2006.

21. Farazi TA, Hoell JI, Morozov P and Tuschl T: MicroRNAs in human cancer. Adv Exp Med Biol 774: 1-20, 2013.

22. Chen L, Wang Q, Wang GD, Wang HS, Huang Y, Liu XM and Cai XH: miR-16 inhibits cell proliferation by targeting IGF1R and the Raf1-MEK1/2-ERK1/2 pathway in osteosarcoma. FEBS Lett 587: 1366-1372, 2013.

23. Zhu H, Wu Y, Zheng W and Lu S: CO-029 is overexpressed in gastric cancer and mediates the effects of EGF on gastric cancer cell proliferation and invasion. Int J Mol Med 35: 798-802, 2015.

24. Xu WG, Shang YL, Cong XR, Bian X and Yuan Z: MicroRNA-135b promotes proliferation, invasion and migration of osteosarcoma cells by degrading myocardin. Int J Oncol 45: 2024-2032, 2014.

25. Sanai N, Alvarez-Buylla A and Berger MS: Neural stem cells and the origin of gliomas. N Engl J Med 353: 811-822, 2005.

26. He L, He X, Lim LP, de Stanchina E, Xuan Z, Liang Y, Xue W, Zender L, Magnus J, Ridzon D, et al: A microRNA component of the p53 tumour suppressor network. Nature 447: 1130-1134, 2007.

27. Seike M, Goto A, Okano T, Bowman ED, Schetter AJ, Horikawa I, Mathe EA, Jen J, Yang P, Sugimura H, et al: MiR-21 is an EGFRregulated anti-apoptotic factor in lung cancer in never-smokers. Proc Natl Acad Sci USA 106: 12085-12090, 2009.
28. Lal A, Navarro F, Maher CA, Maliszewski LE, Yan N, O'Day E, Chowdhury D, Dykxhoorn DM, Tsai P, Hofmann O, et al: miR-24 Inhibits cell proliferation by targeting E2F2, MYC, and other cell-cycle genes via binding to 'seedless' 3'UTR microRNA recognition elements. Mol Cell 35: 610-625, 2009.

29. Gupta PB, Chaffer CL and Weinberg RA: Cancer stem cells: Mirage or reality? Nat Med 15: 1010-1012, 2009.

30. Visvader JE and Lindeman GJ: Cancer stem cells in solid tumours: Accumulating evidence and unresolved questions. Nat Rev Cancer 8: 755-768, 2008.

31. Siclari VA and Qin L: Targeting the osteosarcoma cancer stem cell. J Orthop Surg 5: 78, 2010.

32. Chumsri S and Burger AM: Cancer stem cell targeted agents: Therapeutic approaches and consequences. Curr Opin Mol Ther 10: 323-333, 2008

33. Costello RT, Mallet F, Gaugler B, Sainty D, Arnoulet C, Gastaut JA and Olive D: Human acute myeloid leukemia CD34 $/$ CD38 progenitor cells have decreased sensitivity to chemotherapy and Fas-induced apoptosis, reduced immunogenicity, and impaired dendritic cell transformation capacities. Cancer Res 60: 4403-4411, 2000.

34. Dean M, Fojo T and Bates S: Tumour stem cells and drug resistance. Nat Rev Cancer 5: 275-284, 2005.

35. Deng S, Calin GA, Croce CM, Coukos G and Zhang L: Mechanisms of microRNA deregulation in human cancer. Cell Cycle 7: 2643-2646, 2008.

36. Calin GA, Dumitru CD, Shimizu M, Bichi R, Zupo S, Noch E, Aldler H, Rattan S, Keating M, Rai K, et al: Frequent deletions and down-regulation of micro-RNA genes miR 15 and miR16 at 13q14 in chronic lymphocytic leukemia. Proc Natl Acad Sci USA 99: 15524-15529, 2002.

37. Calin GA, Ferracin M, Cimmino A, Di Leva G, Shimizu M, Wojcik SE, Iorio MV, Visone R, Sever NI, Fabbri M, et al: A MicroRNA signature associated with prognosis and progression in chronic lymphocytic leukemia. N Engl J Med 353: 1793-1801, 2005.

38. Lu J, Getz G, Miska EA, Alvarez-Saavedra E, Lamb J, Peck D, Sweet-Cordero A, Ebert BL, Mak RH, Ferrando AA, et al: MicroRNA expression profiles classify human cancers. Nature 435: 834-838, 2005.

39. Johnson SM, Grosshans H, Shingara J, Byrom M, Jarvis R, Cheng A, Labourier E, Reinert KL, Brown D and Slack FJ: RAS is regulated by the let-7 microRNA family. Cell 120: 635-647, 2005.

40. Saito Y, Liang G, Egger G, Friedman JM, Chuang JC, Coetzee GA and Jones PA: Specific activation of microRNA-127 with downregulation of the proto-oncogene $B C L 6$ by chromatin-modifying drugs in human cancer cells. Cancer Cell 9: 435-443, 2006.

41. Wu Q, Jin H, Yang Z, Luo G, Lu Y, Li K, Ren G, Su T, Pan Y, Feng B, et al: MiR-150 promotes gastric cancer proliferation by negatively regulating the pro-apoptotic gene EGR2. Biochem Biophys Res Commun 392: 340-345, 2010.

42. Ribas J and Lupold SE: The transcriptional regulation of miR-21, its multiple transcripts, and their implication in prostate cancer. Cell Cycle 9: 923-929, 2010.

43. Chow TF, Mankaruos M, Scorilas A, Youssef Y, Girgis A, Mossad S, Metias S, Rofael Y, Honey RJ, Stewart R, et al: The miR-17-92 cluster is over expressed in and has an oncogenic effect on renal cell carcinoma. J Urol 183: 743-751, 2010. 